\title{
Output Voltage Ripple Analysis and Design Considerations of Intrinsic Safety Flyback Converter Based on Energy Transmission Modes
}

\author{
Wei $\mathrm{Hu}^{*}$, Fangying Zhang ${ }^{\dagger}$, Yawu Xu* ${ }^{*}$, and Xinbing Chen ${ }^{*}$ \\ ${ }^{*}$ Lab Center, Guangzhou University, Guangzhou, China
}

\begin{abstract}
For the purpose of designing an intrinsic safety Flyback converter with minimal output voltage ripple based on a specified output current, this paper first classified the energy transmission modes of the system into three sorts, namely, the Complete Inductor Supply Mode-CCM (CISM-CCM), the Incomplete Inductor Supply Mode-CCM (IISM-CCM) and the Incomplete Inductor Supply Mode-DCM (IISM-DCM). Then, the critical secondary self-inductance assorting the three modes are deduced and expressions of the output voltage ripples (OVR) are presented. For a Flyback converter with constant loads and switching frequency, it is shown that the output voltage ripple in the CISM-CCM is the smallest and that it has no relationship with the secondary self-inductance. Otherwise, the OVR of the other two modes are bigger than the previously mentioned one. It is concluded that the critical inductance between the CISM-CCM and the IISM-CCM is the minimal secondary self-inductance to ensure the smallest output voltage ripple. At last, a design method to guarantee the minimum OVR within the scales of the input voltage and load are analyzed, and the minimum secondary self-inductance is proposed to minimize the OVR. Simulations and experiments are given to verify the results.
\end{abstract}

Key words: Critical inductance, Intrinsic safety flyback converter, Magnetizing inductance, Maximum output voltage ripple, Minimum output voltage ripple

\section{INTRODUCTION}

DC-DC converters have been widely applied in industrial circles [1]. In some special cases, such as coal mines, underground and chemical engineering, the specific requirements of anti-explosive and anti-spark should be considered when designing a converter. For these reasons, much attention has been paid in the research to intrinsic safety converters.

Intrinsic safety is defined as a protection technique based on the restriction of electrical energy to a level that is too low to ignite an explosive atmosphere [2]. Since the stored energy varies directly with the reactive component values at specific output voltages, the key points for designing an appropriate intrinsic safety system are making use of the minimum inductors and capacitors under conditions that satisfy the

Manuscript received Mar. 2, 2014; accepted Jun. 20, 2014

Recommended for publication by Associate Editor Honnyong Cha.

${ }^{\dagger}$ Corresponding Author: zfy@gzhu.edu.cn

Tel: +86-020-39366400, Guangzhou University

*Lab Center, Guangzhou University, China requirements of the output voltages and their ripples [3]. Although, increasing the switching frequency could minimize the size and weight of the electric components, it would lead to some drawbacks at very high switching speeds, such as increasing of the switching losses, switching noise and so on. As a result, researchers focus on analyzing the operating modes and output voltage ripples as well as the relationship between the circuit parameters and trying to improve the intrinsic safety of the converter by choosing appropriate components. Thus, for a DC-DC converter with specific output voltage (or current) and OVR, finding the minimum number of inductors and capacitors will be the most important task in a system design procedure.

Researchers are dedicated to the analysis the OVR of DC-DC converters [4]. Several recent works had focused on the OVR of intrinsic safety non-isolation DC-DC converters, such as Buck [5], Boost [3], [6], Buck-Boost [7] and SEPIC converters [8]. They suggest that as the inductance value changes, there will be new energy transfer modes (ETM) in the system, i.e., CISM and IISM. By analyzing the new operation modes, it is shown that the OVR closely relates to the 
inductance. Computing the amplitude of the OVR has played a very important role in the design of converters. Based on the analysis of the correlations between the ETM and the output voltage ripples, the performances of intrinsic safety converters are further improved by properly designing the parameters of the circuit components.

When compared to the Buck-Boost converters and so on, the Flyback converter has the advantages of input/output isolation, low cost and a versatile conversion ratio, which are more appropriate for application under explosive conditions. However, the majority of the studies carried out on converters are concentrated with topology reconstructions and industrial applications, see [9]-[14]. They never focus on the design considerations of intrinsic safety Flyback converters. The mechanism of the ETM is neglected, which leads to inaccurate conclusions in terms of the operation modes for the system.

When analyzing the operation modes of Flyback converters, they are traditionally separated it into continuous conduction mode (CCM) and discontinuous conduction mode (DCM) [1], [15]. It is generally deemed that the OVR in CCM operation converter is irrelevant to the magnetizing inductance and secondary self-inductance. Nevertheless, the theoretical analysis and experimental results manifest that this conclusion is only correct under certain conditions. However, in some cases, the OVR of a Flyback converter increases significantly while the magnetizing inductance decreases, which is inconsistent with the existing theoretical analysis results.

Traditionally, a Flyback converter is an isolated buck-boost converter [16], [17]. Taking the turns ratio into account as an extra degree-of-freedom, both the output voltage and the current are affected. This leads the different design methods to assure the minimum OVR from the basic buck-boost converter, which needs to be thoroughly analyzed. The ETM of the converter only has a relationship with the secondary self-inductance. As a result, the connection between the output current and the secondary winding should be confirmed by mathematical calculations. Furthermore, the optimal parameters of the circuit components will be obtained.

For the purpose of classifying the ETM of the object, the energy transfer process and the OVR in Flyback converters are analyzed in depth so as to design an intrinsic safety system. The relationship between the ETM, the OVR and the component parameters are deduced, and the minimum secondary self-inductance to guarantee the minimum OVR is obtained. These are of great use for transformer and converter miniaturizations.

This paper is organized as follows. In the next Section, the ETM of the intrinsic safety Flyback converter is analyzed. The critical secondary self-inductances between CCM and DCM, as well as the CISM-CCM and IISM-CCM are derived in Section III. Section IV provides the OVR of the different operational modes belonging to the Flyback converter. The Maximum and Minimum OVRs (MOVRs) of the regulator are presented in
Section V. After that, the design considerations for choosing proper circuit parameters are discussed in Section VI. Simulation and experimental results are given in Section VII. At last, some conclusions are given in Section VIII.

\section{ETM OF FLYBACK CONVERTER}

The topology of a Flyback converter is shown in Fig. 1(a). Assuming that the Flyback transformer has a $k=W_{1} / W_{2}$ turns ratio and a leakage inductance $L_{e}$, let the magnetizing inductance and secondary self-inductance be $L_{M}$ and $L_{s}$, respectively. Then, the following equations will be obtained based on the electrical model of the transformer [18]:

$$
\begin{aligned}
& L_{s}=\frac{L_{M}}{k^{2}}, \\
& V_{i n n}=\eta V_{i n},
\end{aligned}
$$

where, $V_{i n}$ and $V_{i n n}$ denote the input voltage and secondary self-inductance voltage, respectively, and $\eta=\frac{L_{M}}{L_{e}+L_{M}}$.

The energy transfer mode is complicated when switch $\mathrm{S}$ turns off and the secondary winding connects to the circuit. Now the ETM is closely related to the quantity of the secondary self-inductance. There will be a critical inductance $L_{s C}$. When $L_{s}>L_{s C}$, the converter operates in CCM, and when $L_{s}<L_{s C}$, the converter operates in DCM. Diagrams of the different operation modes are shown in Fig. 1(b), (c), (d) and (e).

\section{A. ETM of DCM Flyback Converter}

While the converter operates in DCM, the inductance currents and the output voltage waveforms are sketched in Fig. 2(c). The ETM may be separated into three subintervals as the switch turns off at time instant $t_{1}$.

First subinterval $\left(t_{1} \sim t_{2}\right)$ : Only the secondary winding inductor discharges energy in this stage. Fig. 1(c) shows the equivalent circuit. The inductance $L_{s}$ supplies energy to the output capacitor and the load resistance, which leads to an increase of the capacitor voltage. This stage lasts until time instant $t_{2}$. Meanwhile $i_{S}$ declines to $I_{O}$.

Second sub-interval $\left(t_{2} \sim t_{3}\right)$ : This stage begins when $\left(i_{s}<I_{O}\right)$. In this case, both the secondary self-inductance and the capacitor provide energy for the load resistance. The equivalent circuit is shown in Fig. 1(d).

Third sub-interval $\left(t_{3} \sim t_{4}\right)$ : This time sub-interval begins at the moment $i_{s}$ reaches zero. Since the next period has not come, there is only the capacitor supplying energy to the load. Fig. 1 (e) shows the equivalent schematic. 


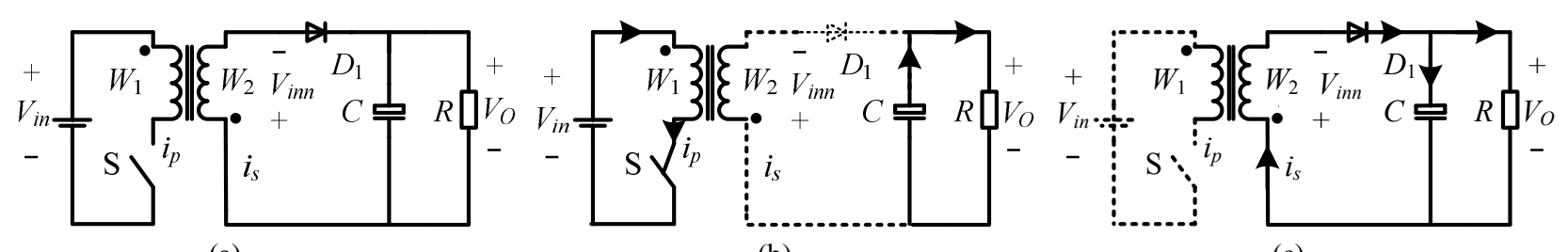

(a)

(b)

(c)

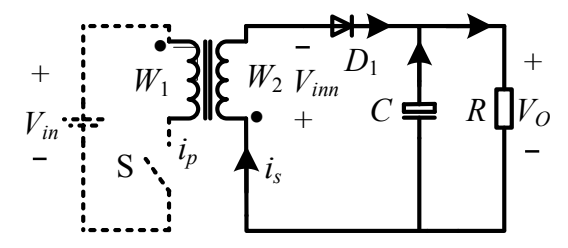

(d)

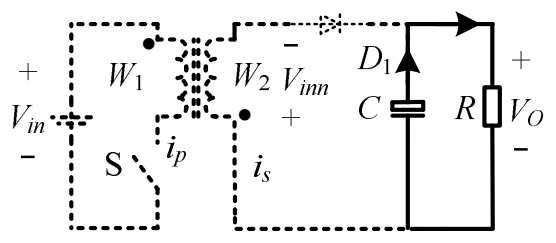

(e)

Fig. 1. Operation modes of Flyback converter. (a) Flyback converter. (b) Switch S connects. (c) Flyback converter in CISM-CCM. (d) Flyback converter in IISM-CCM. (e) Flyback converter in IISM-DCM.

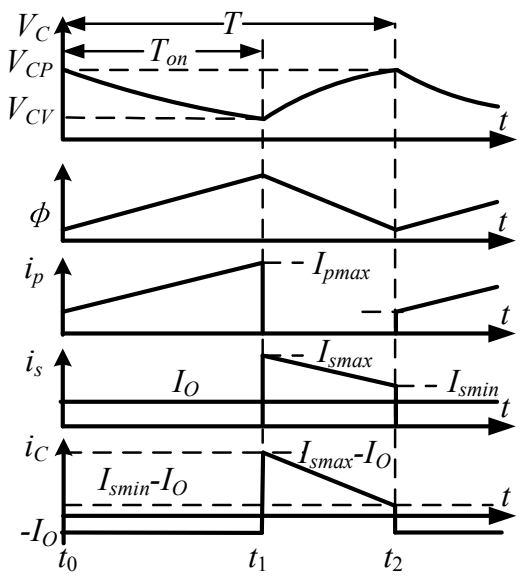

(a)

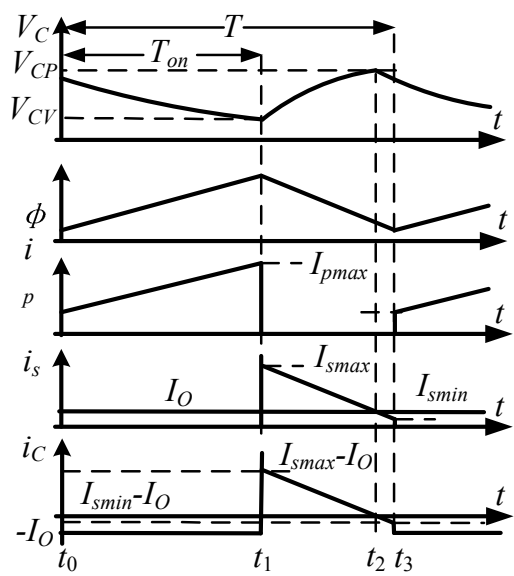

(b)

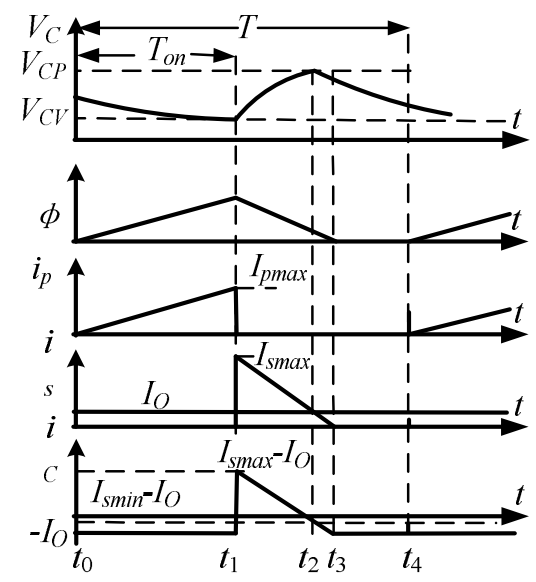

(c)

Fig. 2. Output voltage, magnetic flux, primary inductor current of transformer, secondary inductor current of transformer and capacitor current waveforms of Flyback converter. (a) The CISM-CCM mode. (b) The IISM-CCM mode. (c) The IISM-DCM mode.

It is evident that for a Flyback converter performing in DCM, the ETM is divided into the three parts after the switch disconnects, i.e,: the inductance supplies energy, the inductance and capacitor supply energy at the same time, and the capacitor supplies energy.

\section{B. ETM of CCM Flyback Converter}

In the case of CCM operation, according to a comparison between $I_{s \min }$ (the minimum magnitude of the secondary winding current $i_{s}$ ) and $I_{O}$, the operation processes of the converter can be further divided into two kinds of energy transfer modes. When $I_{s \min }>I_{O}$, it is called CISM-CCM; when $I_{s \min }<I_{O}$, it is called IISM-CCM. The concrete analyses are as follows:

CISM-CCM: In this case, $I_{s \min }>I_{O}$. The switch tube $\mathrm{S}$ is blocked, the secondary winding inductor supplies energy both to the load and to the capacitor. Fig. 1(c) shows the equivalent diagram. The waveforms of the second-side inductor current and the capacitor voltage are portrayed in Fig. 2(a).
IISM-CCM: In this case, $I_{s \min }<I_{O}$. The waveforms, including the capacitor voltage, inductor currents and magnetic flux, are depicted in Fig. 2(b). The ETM of the converter can be separated into two parts. The first part is that the inductor completely charges the capacitor and load, the equivalent circuit of which is shown in Fig. 1(c). In this case, $i_{s}>I_{O}$, the capacitor voltage increases linearly. The second part is that the secondary winding inductor and the output capacitor simultaneously supply current for the load. Fig. 1(d) shows the equivalent scheme. At this moment, $i_{s}<I_{O}$, and the voltage of the capacitor starts to go down and will not stop until the next clock pulse signal comes.

It is clear that the critical condition of the Flyback converter operates between CISM-CCM and IISM-CCM is $I_{s \min }=I_{O}$.

\section{DERIVATION OF THE CRITICAL INDUCTOR}

The boundary between the CCM and DCM is specified by the critical inductance $L_{s C}$ with regard to $L_{s}$. By considering 
the output current $I_{O}$ and the secondary winding current $I_{s}$, the CCM mode is divided into two operational modes, namely, CISM-CCM and IISM-CCM. The borderline of these two operational modes is defined by the critical inductance $L_{s k}$.

\section{A. Critical inductance $L_{s C}$}

As for the Flyback converter, the following equations are always workable with the leakage inductance:

$$
d=\frac{T_{o n}}{T}=\frac{k V_{O}}{k V_{O}+\eta V_{\text {in }}} .
$$

The critical inductance of CCM and DCM is as follows:

$$
L_{s C}=\frac{R}{2 f}(1-d)^{2}=\frac{R \eta^{2} V_{i n}^{2}}{2 f\left(k V_{O}+\eta V_{i n}\right)^{2}} .
$$

\section{B. Critical inductance $L_{s k}$}

The minimum secondary inductor current can be written as:

$$
I_{s \min }=I_{O}\left[\frac{1}{1-d}-\frac{R}{2 L_{s} f}(1-d)\right] .
$$

It is clear that the critical condition in CISM-CCM and IISM-CCM is $I_{s \min }=I_{O}$, which leads to:

$$
L_{s k}=\frac{R}{2 d f}(1-d)^{2}=\frac{R \eta^{2} V_{i n}^{2}}{2 k f V_{O}\left(k V_{O}+\eta V_{i n}\right)} .
$$

Since $d \leq 1$, it is obtained that $L_{s k}>L_{s C}$.

According to the forgoing analysis, the operation mode boundary of the entire system is illustrated in Table I.

\section{OVR OF THE FLYBACK CONVERTER}

The amplitudes of the OVRs of the Flyback converter in different operation modes will be calculated in detail.

\section{A. Calculation of the OVR in CISM-CCM}

Obviously, in the time-subinterval $T_{o n}$, the output voltage is equal to the capacitor voltage. From Fig. 2 (a), it can be seen that:

$$
V_{C P}=V_{C V}-\frac{1}{C} \int_{0}^{T_{o n}} i_{C}(t) d t
$$

Then, the OVR can be expressed as:

$$
V_{P P}^{C I S M-C C M}=V_{C P}-V_{C V}=-\frac{1}{C} \int_{0}^{T_{o n}} i_{C}(t) d t .
$$

In the time frame of $T_{o n}$, the capacitor current is $-I_{O}$, and the following can be obtained:

$$
V_{P P}^{C I S M-C C M}=\frac{d T I_{O}}{C} .
$$

Then, the following is obtained:

$$
V_{P P}^{C I S M-C C M}=\frac{k V_{O}^{2}}{\left(\eta V_{\text {in }}+k V_{O}\right) f C R} .
$$

From Eq. (10), it can be concluded that the OVR of the converter has no connection with the secondary self-inductance in CISM-CCM operation.
TABLE I

Operation Mode BOUndary OF THE CONVERTER

\begin{tabular}{cc}
\hline Inductance Region & Operation Mode \\
\hline$L_{s}>L_{s k}$ & CISM-CCM \\
$L_{s C}<L_{s}<L_{s k}$ & IISM-CCM \\
$L_{s}<L_{s C}$ & IISM-DCM \\
\hline
\end{tabular}

\section{B. Calculation of the OVR in IISM-CCM}

The OVR in this mode is determined by the voltage waveform in the time sub-interval $\left(t_{1}, t_{2}\right)$ when switch $\mathrm{S}$ is turned off, as can be seen in Fig. 2 (b). Obviously, the following is obtained:

$$
i_{C}(t)=i_{s}(t)-I_{O} .
$$

In addition, the equations of the secondary winding inductor current can be written as:

$$
i_{s}=-\frac{V_{O}}{L_{s}} t+I_{s \max } .
$$

The application of Eq. (12) to (11), results in:

$$
i_{C}(t)=-\frac{V_{O}}{L_{s}} t+I_{s \max }-I_{O} .
$$

At the time instant $t=t_{2}, i_{C}\left(t_{2}\right)$ reaches zero. As a result, $i_{s}\left(t_{2}\right)=I_{O}$. Taking $t_{2}$ as the time benchmark leads to:

$$
\Delta t=t_{2}-t_{1}\left(I_{s \max }-I_{O}\right) \frac{L_{s}}{V_{O}} .
$$

Consequently, the OVR in this time sub-interval can be written as:

$$
V_{P P}^{I I S M-C C M}=\frac{1}{C} \int_{0}^{\Delta t}\left(I_{s \max }-I_{O}-\frac{V_{O}}{L_{s}} t\right) d t .
$$

Substitution of the above equations into Eq. (15) yields:

$$
V_{P P}^{I I S M-C C M}=\frac{L_{s} V_{O}}{2 C}\left[\frac{k V_{O}}{\eta R V_{i n}}+\frac{\eta V_{i n}}{2 L_{s} f\left(k V_{O}+\eta V_{i n}\right)}\right]^{2} .
$$

From Eq. (16) the OVR is connected with the value of the secondary self-inductance $L_{s}$. The ripple has a relationship with the turns ratio $k$ of the transformer. In order to simplify the analysis and with a view to the proportional relationship between $L_{s}$ and $k$, only $L_{s}$ is discussed in the following.

Calculating the first partial derivative of Eq. (16) yields:

$$
L_{s}=\frac{R \eta^{2} V_{i n}^{2}}{2 k f V_{O}\left(k V_{O}+\eta V_{i n}\right)}=L_{s K} .
$$

Calculating the second partial derivative of Eq. (16) leads to:

$$
\frac{\partial^{2} V_{P P}}{\partial L_{s}^{2}}=\frac{\eta^{2} V_{O} V_{i n}^{2}}{4 C f^{2} L_{s}^{3}\left(k V_{O}+\eta V_{i n}\right)^{2}} .
$$

From Eqs. (16), (17) and (18), it is know that when $L_{s}<L_{s k}$, $\partial V_{P P} / \partial L_{s}<0$. Therefore, the voltage ripple $V_{P P}^{I I S M-C C M}$ 
decreases with an increase of the inductance $L_{s}$ in $L_{s C}<L_{s}<L_{s k}$. By putting Eqs. (17) and (4) into Eq. (16), the minimum and maximum of $V_{P P}^{I I S M-C C M}$ are obtained as follows:

$$
\begin{gathered}
V_{P P, \min }^{I I S M-C C M}=\frac{k V_{O}^{2}}{\left(\eta V_{i n}+k V_{O}\right) f C R}=V_{P P}^{(C I S M-C C M)}, \\
V_{P P, \max }^{I I S M-C C M}=\frac{\eta V_{O} V_{i n}\left(2 k V_{O}+\eta V_{i n}\right)}{4 f C\left(k V_{O}+\eta V_{i n}\right)^{2}} .
\end{gathered}
$$

\section{Calculation of the OVR in IISM-DCM}

The maximum value of the primary winding inductor current is the current climbing value in the time interval $T_{o n}=d T$, which is the duration when switch $\mathrm{S}$ connects. Because $i_{p}\left(t_{0}\right)$ is equal to zero at the beginning of every switching period, the following is obtained:

$$
I_{p \max }=\frac{d T \eta V_{i n}}{L_{M}}=\frac{I_{s \max }}{k} .
$$

The following equation is true when the Flyback converter works in DCM:

$$
d=\frac{V_{O}}{\eta V_{\text {in }}} \sqrt{\frac{2 L_{M} f}{R}}=\frac{k V_{O}}{\eta V_{\text {in }}} \sqrt{\frac{2 L_{s} f}{R}} .
$$

Putting Eqs. (13), (14), (17) and (21) into Eq. (22), leads to:

$$
V_{P P}^{I I S M-D C M}=\frac{L_{s} V_{O}}{2 C}\left(\sqrt{\frac{2}{R L_{s} f}}-\frac{1}{R}\right)^{2} .
$$

Calculate the partial derivative with respect to $L_{s}$ on Eq.(19) and let it be zero, the result is:

$$
L_{s D}=\frac{2 R}{f} .
$$

Comparing Eq. (4) with (20), it can be seen that:

$$
L_{s D}=\frac{4}{(1-d)^{2}} L_{C}>L_{C} \text {. }
$$

Then, calculate the partial derivative with respect to $L_{s}$ in Eq.(19), and the following is obtained:

$$
\frac{\partial^{2} V_{P P}^{I I S M-D C M}}{\partial L_{s}^{2}}=\frac{L^{-3 / 2}}{4 C f R} \sqrt{\frac{2 f}{R}}>0 .
$$

Combine Eqs. (19), (20), (21) and (22). It can then be concluded that when $L_{s}<L_{S D}, V_{P P}^{I I S M-D C M}$ decreases with an increase in the value of the inductance. However, when in IISM-DCM, there is $L_{s}<L_{s C}<L_{s D}$. Combine Eq. (4) into (19), and consider Eq. (3) when $L_{S}=L_{s C}$. Then the minimum value of $V_{P P}^{I I S M-D C M}$ can be obtained:

$$
V_{P P, \min }^{I I S M-D C M}=\frac{\eta V_{O} V_{i n}\left(2 k V_{O}+\eta V_{i n}\right)}{4 f C\left(\eta V_{i n}+k V_{O}\right)^{2}}=V_{P P, \max }^{I I S M-C C M} .
$$

The above analysis demonstrates that the OVR of a Flyback converter operating in CISM-CCM is the smallest, and that the

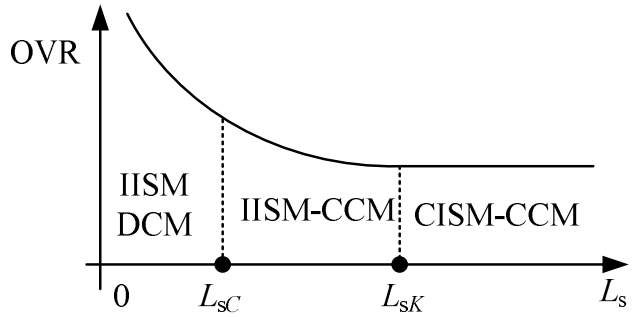

Fig. 3. Variations of OVR based on secondary self-inductance.

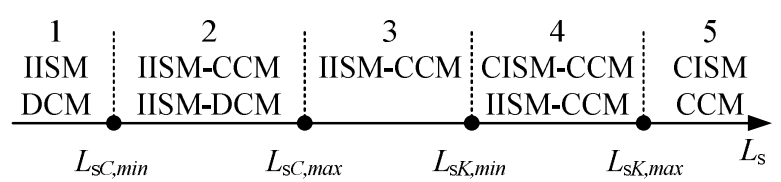

Fig. 4. Classifications of operation modes under $L_{s C \text {, max }}<L_{s K \text {, min }}$ condition.

amplitude is irrelevant to the inductance. Meanwhile, the OVRs in IISM-CCM and IISM-DCM are much bigger and increase with a decrease in the inductance. Therefore, the OVR decreases as the secondary self-inductance increases, and reaches its minimum value when $L_{s}=L_{s k}$. In other words, the critical secondary self-inductance $L_{s k}$ is the minimum one which makes the OVR of the system become the smallest in the whole operation region. The variations of the OVR based on the secondary self-inductance are sketched in Fig. 3.

\section{MAXimum AND Minimum OUTPUT VoltaGE RIPPLES}

According to the former analysis, when $V_{\text {in }}$ and load $R$ decrease, the value of the OVR increases, and vice versa. In comparison to the variation of the critical inductance, the operation areas of the system can be separated into five regions. Generally speaking, under the conditions of $L_{s C \text {, max }}<L_{s K \text {, min }}$ or $L_{s C \text {, max }}>L_{s K \text {, min }}$, the operation areas can be classified into two regions.

\section{A. $L_{s C \text {, max }}<L_{s K, \min }$}

In the case of $L_{s C \text {, max }}<L_{s K \text {,min }}$, the operation areas can be separated into five parts. The typical classifications are portrayed in Fig. 4.

1) IISM-DCM when $L_{s}<L_{s K \text {,min }}$ : In the first operation area, the minimum value of MOVR is used to get under the $L_{s}=L_{s C \text {, min }}$ condition. Taking Eq. (23) into account yields:

$$
\min \left\{V_{P P 1, \max }^{I I S M-D C M}\right\}=\frac{L_{s C, \min } V_{O}}{2 C}\left(\sqrt{\frac{2}{R_{\min } L_{S C, \min } f}}-\frac{1}{R_{\min }}\right)^{2} .
$$

2) If $L_{s C \text {, } \min }<L_{s}<L_{s C \text {,max }}$ : In this area, as the system operates in IISM-CCM. Calculating the minimum magnitude of MOVR by Eq. (16) yields:

$$
\min \left\{V_{P P 2, \max }^{I I S M-C C M}\right\}=\frac{L_{s C, \max } V_{O}}{2 C}
$$




$$
\left(\frac{k V_{O}}{\eta R_{\min } V_{i n, \text { min }}}+\frac{\eta V_{i n, \text { min }}}{2 L_{s C, \text { max }} f\left(k V_{O}+\eta V_{i n, \min }\right)}\right)^{2} .
$$

The maximum magnitude of MOVR in this area is obtained as the system operates in IISCM-DCM. Therefore, MOVR in this sub-part is given by:

$$
\max \left\{V_{P P 2, \max }^{I I S M-D C M}\right\}=\frac{L_{s C, \min } V_{O}}{2 C}\left(\sqrt{\frac{2}{R_{\min } L_{s C, \min } f}}-\frac{1}{R_{\min }}\right)^{2} .
$$

3) If $L_{s C, \max }<L_{s}<L_{s K, \min }$ : In this case, the system only operates in IISM-CCM. Calculating the minimum and maximum magnitude of MOVR by Eq. (16) yields:

$$
\begin{gathered}
\min \left\{V_{P P 3, \max }^{I I S M-C C M}\right\}=\frac{L_{s K, \text { min }} V_{O}}{2 C} \\
\left(\frac{k V_{O}}{\eta R_{\min } V_{i n, \text { min }}}+\frac{\eta V_{i n, \text { min }}}{2 L_{s K, \text { min }} f\left(k V_{O}+\eta V_{i n, \text { min }}\right)}\right)^{2} . \\
\max \left\{V_{P P 3, \max }^{I I S M-C C M}\right\}=\frac{L_{s K, \text { max }} V_{O}}{2 C} \\
\left(\frac{k V_{O}}{\eta R_{\text {min }} V_{i n, \text { min }}}+\frac{\eta V_{i n, \text { min }}}{2 L_{s K, \text { max }} f\left(k V_{O}+\eta V_{i n, \text { min }}\right)}\right)^{2} .
\end{gathered}
$$

Considering Eqs. (19) and (31), the minimum value of MOVR leads to:

$$
\min \left\{V_{P P 3, \max }^{I I S M-C C M}\right\}=\frac{k V_{O}^{2}}{\left(\eta V_{i n, \min }+k V_{O}\right) f C R_{\text {min }}} .
$$

4) If $L_{s K, \min }<L_{s}<L_{s K, \max }$ : In this case, the system only operates in IISM-CCM. Calculating the minimum and maximum magnitude of MOVR by Eq. (16) yields:

$$
\begin{aligned}
\max \left\{V_{P P 4, \max }^{I I S M-C C M}\right\} & =\frac{L_{s K, \text { min }} V_{O}}{2 C} \\
& \left(\frac{k V_{O}}{\eta R_{\min } V_{i n, \min }}+\frac{\eta V_{i n, \text { min }}}{2 L_{s K, \text { min }} f\left(k V_{O}+\eta V_{i n, \text { min }}\right)}\right)^{2} .
\end{aligned}
$$

On the other hand, if the converter operates in CISM-CCM, the minimum magnitude of MOVR is given by:

$$
\min \left\{V_{P P 4, \max }^{C I S M-C C M}\right\}=\frac{k V_{O}^{2}}{\left(\eta V_{i n, \max }+k V_{O}\right) f C R_{\min }} .
$$

Taking Eqs. (19) and (34) into consideration, the maximum OVR will be shown as:

$$
\max \left\{V_{P P 4, \max }^{C I S M-C C M}\right\}=\frac{k V_{O}^{2}}{\left(\eta V_{i n, \min }+k V_{O}\right) f C R_{\min }} .
$$

5) If $L_{s}>L_{s K, \max }$ : In this case, the system only operates in CISM-CCM. Calculating Eq. (10) leads to:

$$
V_{P P 5, \max }^{C I S M-C C M}=\frac{k V_{O}^{2}}{\left(\eta V_{i n, \min }+k V_{O}\right) f C R_{\min }} .
$$

As stated above, the sequence of the MOVRs can be shown as:

$$
V_{P P 1, \text { max }}>V_{P P 2, \text { max }}>V_{P P 3, \text { max }}>V_{P P 4, \text { max }}=V_{P P 5, \text { max }} .
$$

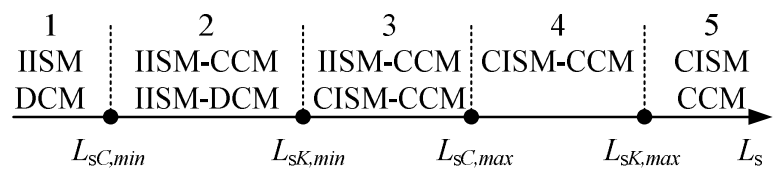

Fig. 5. Different operation modes under $L_{C, \max }>L_{K, \min }$ condition.

B. $L_{s C, \max }>L_{s K, \min }$

The other five classifications of the operation areas under the $L_{S C, \text { max }}>L_{s K, \text { min }}$ condition are typically portrayed as in Fig. 5.

1) IISM-DCM when $L_{s}<L_{s C \text {,min }}$ : Considering Eq. (19), the minimum magnitude of $V_{P P 1, \max }$ in this operation area is shown as follows:

$$
\min \left\{V_{P P 1, \max }^{I I S M-D C M}\right\}=\frac{L_{s C, \min } V_{O}}{2 C}\left(\sqrt{\frac{2}{R_{\min } L_{s C, \min } f}}-\frac{1}{R_{\min }}\right)^{2} .
$$

2) If $L_{s C \text {, min }}<L_{s}<L_{s K, \text { min }}$ : The system performs in either IISM-DCM or IISM-CCM. By calculating Eq. (16), the minimum magnitude of MOVR is shown as:

$$
\begin{aligned}
\min \left\{V_{P P 2, \max }^{I I S M}\right\} & =\frac{L_{s K, \min } V_{O}}{2 C} \\
& \left(\frac{k V_{O}}{\eta R_{\min } V_{i n, \min }}+\frac{\eta V_{i n, \text { min }}}{2 L_{s K, \text { min }} f\left(k V_{O}+\eta V_{i n, \text { min }}\right)}\right)^{2} .
\end{aligned}
$$

Meanwhile, the maximum magnitude of MOVR will be obtained by Eq. (23):

$$
\max \left\{V_{P P 2, \max }^{I I S M-D M}\right\}=\frac{L_{s C, \min } V_{O}}{2 C}\left(\sqrt{\frac{2}{R_{\min } L_{s C, \text { min }} f}}-\frac{1}{R_{\min }}\right)^{2} .
$$

3) If $L_{s K, \min }<L_{s}<L_{s C \text {, } \max }$ : In this case, the Flyback converter performs in either CISM-CCM or IISM-CCM. By calculating Eq. (10), the minimum magnitude of MOVR is shown as:

$$
\min \left\{V_{P P 3, \max }^{C I S M-C C M}\right\}=\frac{k V_{O}^{2}}{\left(\eta V_{i n, \min }+k V_{O}\right) f C R_{\min }} .
$$

Meanwhile, the maximum magnitude of MOVR will be obtained by Eq. (16):

$$
\begin{aligned}
\max \left\{V_{P P 3, \max }^{I I S M-C C M}\right\} & =\frac{L_{s K, \min } V_{O}}{2 C} \\
& \left(\frac{k V_{O}}{\eta R_{\min } V_{i n, \min }}+\frac{\eta V_{i n, \text { min }}}{2 L_{s K, \text { min }} f\left(k V_{O}+\eta V_{i n, \min }\right)}\right)^{2} .
\end{aligned}
$$

Taking Eqs. (19) and (27) into consideration, the minimum magnitude of MOVR in this area is given by:

$$
V_{P P 3, \max }^{C I S M-C C M}=\frac{k V_{O}^{2}}{\left(\eta V_{i n, \text { min }}+k V_{O}\right) f C R_{\text {min }}} .
$$

4) If $L_{s C, \max }<L_{s}<L_{s K, \max }$ : In this case, the system only operates in CISM-CCM. The MOVR is obtained as follows:

$$
V_{P P 4, \text { max }}^{C I S M-C C M}=\frac{k V_{O}^{2}}{\left(\eta V_{i n, \text { min }}+k V_{O}\right) f C R_{\text {min }}} .
$$

5) If $L_{s}>L_{s K \text {, max }}$ : In this case, the system only operates in 
CISM-CCM. The expression of MOVR is as follows:

$$
V_{P P 5, \max }^{C I S M-C C M}=\frac{k V_{O}^{2}}{\left(\eta V_{i n, \min }+k V_{O}\right) f C R_{\min }} .
$$

Based on the former analyses, the sequence of the MOVRs can be shown as:

$$
V_{P P 1, \text { max }}>V_{P P 2, \text { max }}>V_{P P 3, \text { max }}>V_{P P 4, \text { max }}=V_{P P 5, \text { max }} .
$$

\section{DESIGN CONSIDERATIONS OF THE FLYBACK CONVERTER}

As for voltage controlled Flyback converters, the magnitude of the OVR is closely related to the magnetizing inductance and loads at a specific switching period. In most cases, the smaller the OVR is, the better electrical characteristic becomes. According to the expression of the OVR in different operating modes, it is reversely related to $L_{M}, C$, and $f$. To ensure the minimum magnitude of the OVR in industrial applications, the switching period of the converter must be decreased. However, owing to the limitations of the manufacturing technology level, the switching frequency is finite. Meanwhile, along with the short switching periods are the high costs and switching losses. As a result, deductions of the minimum OVR with appropriate capacitor and magnetizing inductance are of great importance.

\section{A. Suggested Inductance in CCM Operation}

According to Eqs. (37) and (46), the minimum OVR is ensured when the converter operates in CISM-CCM, which means the inductance $L_{s}$ should be designed larger than $L_{s K, \max }$. Nevertheless, this is not suitable for industrial applications because the high duty cycle of the Flyback converter would leads to lower efficiency and so on. Therefore, $L_{s}$ should be chosen so that it is lower than $L_{s K, \max }$. It is clear that the minimum OVR was obtained when the condition $L_{s}>L_{s K \text {,min }}$ was ensured:

$$
\min \left\{V_{P P, \max }\right\}=\frac{k V_{O}^{2}}{\left(\eta V_{i n, \min }+k V_{O}\right) f C R_{\min }} .
$$

If the condition $L_{s}>L_{s K, \text { min }}$ is meet, according to Eq. (48), the MOVR is independent from the magnetizing inductance. As a result, the minimum secondary self-inductance $L_{s K, \min }$ is the right one to ensure the minimum MOVR in IISM-CCM.

$$
L_{s \text { min }}=L_{s K, \text { min }}=\frac{R_{\text {min }} \eta^{2} V_{i n, \text { min }}^{2}}{\left(\eta V_{i n, \text { min }}+k V_{O}\right) 2 k f V_{O}} .
$$

Calculating Eq. (48) leads to the minimum capacitance:

$$
C_{\min }=\frac{k V_{O}^{2}}{\left(\eta V_{i n, \min }+k V_{O}\right) V_{P P, \max } f R_{\min }} .
$$

\section{B. Consideration of the Secondary Self-Inductance in $D C M$}

The OVR has a reverse relation to the load and secondary self-inductance, when the converter is in DCM operation, and independent of input voltage, see Eqs. (28) and (39). Taking
Eqs. (38) and (47) into consideration, the OVR obtains its minimum magnitude as $L_{s}=L_{s C \text {, min }}$. The heavy load would make the peak switching current flow through the secondary winding diode increase remarkably. Therefore, the converter should not operate in DCM with heavy loads at a larger power. However, the condition $L_{s}<L_{s C \text {,max }}$ with a large resistance $R_{\max }$ should be meet if the converter needs to operate in DCM.

\section{SIMULATION AND EXPERIMENTAL RESULTS}

To further validate these results, simulation and experimental tests were carried out. The parameters chosen for the intrinsic safety Flyback converter are as follows: $V_{\text {in }}=20 \sim 30 \mathrm{~V}, \quad V_{O}=12 \mathrm{~V}, \quad R=15 \sim 30 \Omega, f=20 k H_{z}, \quad k=2$, and $V_{P P \text {, min }}=0.1 \mathrm{~V}$. In addition, the primary leakage inductance of the transformer is $L_{e}=0.02 L_{M}$, that is $\eta=0.98$. Considering Eqs. (4), (6), (49) and (50), $L_{s C}=75.8 \mu H, L_{s K, \min }=L_{s K}=137.7 \mu \mathrm{H}$, and $C=200 \mu F$ are obtained.

Fig. 6(a), (b) and (c) show the experimental results of the variations of the OVR versus secondary the self-inductance for specific values of $V_{i n}$ and $R$. Change $L_{s}$ from $50 \mu H$ to $150 \mu \mathrm{H}$ and take the pairs of $R$ and $V_{i n}$ as $\left[R=15 \Omega, V_{i n}=20 \mathrm{~V}\right]$, $\left[R=15 \Omega, \quad V_{i n}=30 \mathrm{~V}\right], \quad\left[R=26 \Omega, \quad V_{i n}=20 \mathrm{~V}\right], \quad$ and $[R=26 \Omega$, $\left.V_{i n}=30 \mathrm{~V}\right]$, separately. The experimental results are depicted in Fig. 6(a). It is clear that the OVR in IISM-CCM and IISM-DCM decrease as the secondary self-inductance increases, while it is kept constant in CISM-CCM.

Since the load resistance is the smallest $(R=15 \Omega)$, with the variation of $L_{s}$, the OVR versus $V_{i n}$ are illustrated in Fig. 6(b). It is clear that the OVR decreases with an increase of the $V_{i n}$.

Choosing the smallest input voltage $\left(V_{i n}=20 \mathrm{~V}\right)$, with the variation of $L_{s}$, the OVR versus $R$ are illustrated in Fig. 6(c). It can be seen from the figure that the OVR decreases with an increase in the load.

According to the above results, a detailed example is shown in the following. The parameters chosen for the intrinsic safety Flyback converter are as follows: $V_{i n}=24 \mathrm{~V}$, $V_{O}=12 \mathrm{~V}, I_{O}=0.8 \mathrm{~A}, R=15 \Omega, f=20 k H_{z}$, and $k=2, C=200 \mu F$. In addition, the primary leakage inductance of the transformer is $L_{e}=0.02 L_{M}$, that is $\eta=0.98$. Considering Eqs. (4), (6) and (49), $L_{s C}=367.4 \mu \mathrm{H}$ and $L_{s K, \min }=L_{s K}=727.6 \mu \mathrm{H}$ are obtained.

Take the magnetizing inductances of the transformers as $L_{M}=986 \mu \mathrm{H}, \quad L_{M}=727 \mu \mathrm{H}, \quad L_{M}=555 \mu \mathrm{H}$ and $L_{M}=329 \mu \mathrm{H}$, separately. Then the secondary self-inductances are, in order, $L_{s}=246.5 \mu \mathrm{H}, L_{s}=181.75 \mu \mathrm{H}, L_{s}=138.75 \mu \mathrm{H}$ and $L_{s}=82.25 \mu \mathrm{H}$, which correspond to CISM-CCM, Critical-Inductance State, IISM-CCM and IISM-DCM, respectively. In addition, the simulation waveforms of the output voltage, the transformer secondary winding and output currents are illustrated in Fig. 7(a), (b), (c) and (d), using the PSIM simulator.

Fig. 7(a) shows that when the system operates in CISM-CCM and the switch disconnects, $i_{s}$ is always greater than $0.8 \mathrm{~A}$. Therefore, the secondary wingding inductor 


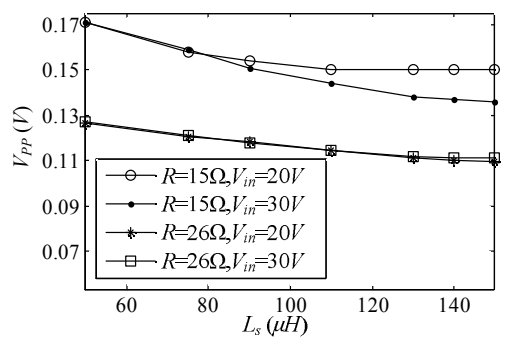

(a)

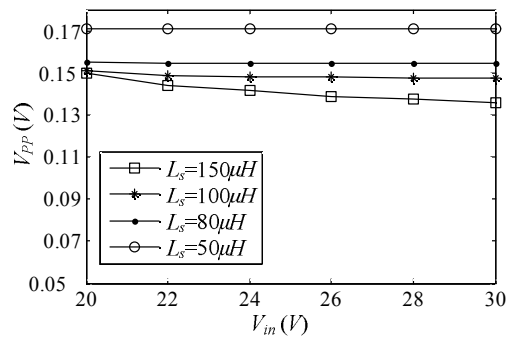

(b)

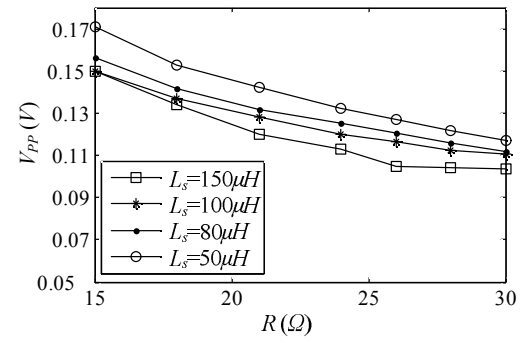

(c)

Fig. 6. Experimental results of variations of OVR. (a) versus $V_{\text {in }}$ and $R$. (b) versus $R$. (c) versus $V_{i n}$.

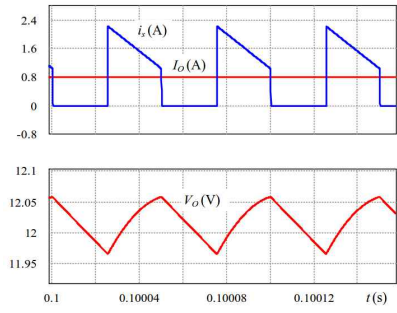

(a)
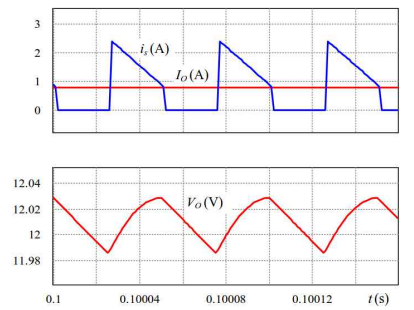

(b)
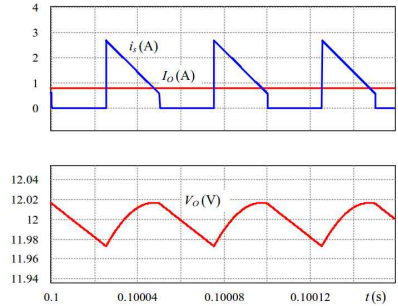

(c)
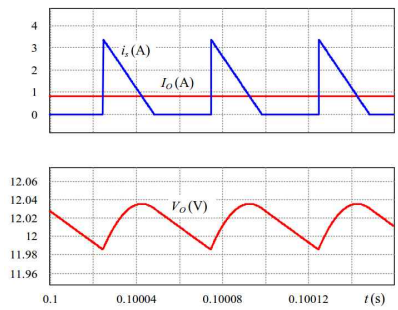

(d)

Fig. 7. Secondary inductor current and output voltage waveforms of Flyback converter in different modes. (a) CISM-CCM. (b) Critical inductance state. (c) IISM-CCM mode. (d) IISM-DCM mode.

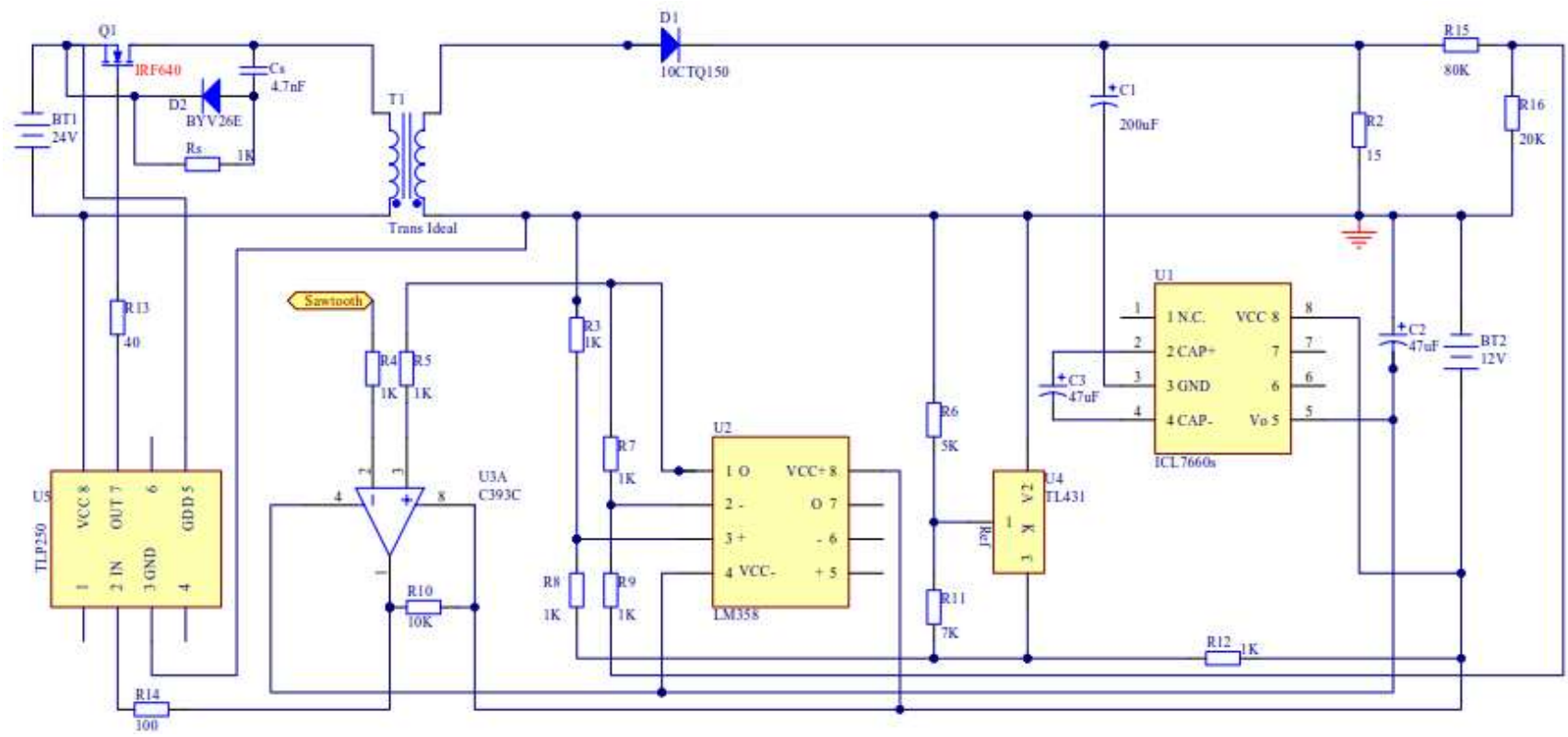

Fig. 8. The circuit diagram of voltage controlled Flyback converter.

charges the capacitor, and the capacitor voltage goes up, and does not go down until the next period. The descend range of the capacitor voltage is only decided by the capacitor. As a result, the OVR in the operation area and the inductance are totally unrelated, and the ripple voltage is smaller.

From Fig. 7(b), it is known that when $L_{s}$ equals $L_{s K}$, the converter operates in the Critical Inductance State, which ensures the Minimum OVR.

From Fig. 7(c), it is known that when the system is in IISM-CCM operation, the switch disconnects, and when $i_{s}$ decreases to less than $0.8 \mathrm{~A}$, the capacitor voltage starts to decrease. Compared with the former, the descend range of the capacitor voltage has an increase and it is relevant to the capacitor and the inductance.

Fig. 7(d) shows that when the converter is in IISM-DCM, the switch blocks and $i_{s}$ decreases to less than 0.8A. Just like the converter in IISM-CCM operation, the capacitor voltage starts to decrease. The difference is that the capacitor voltage needs to experience two descend periods, i.e., $i_{s}<0.8 \mathrm{~A}$ and $i_{s}=0$. It can be seen that the OVR is the best among all of the situations discussed above.

The voltage controlled Flyback converter circuit diagram, as shown in Fig. 8, is set up. The system components are as shown in the figure. It is worth noting that the sawtooth 


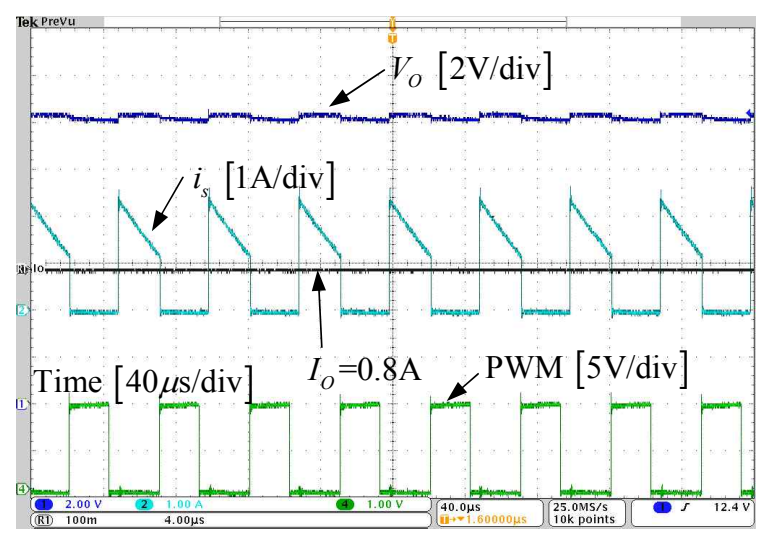

(a)

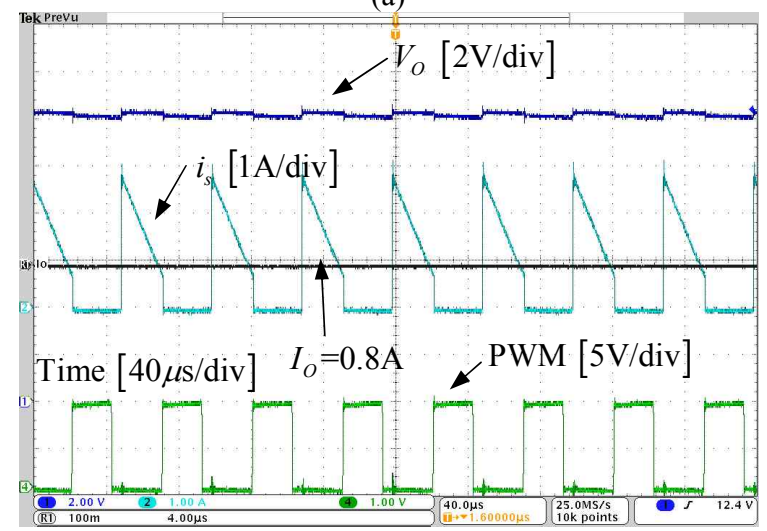

(c)

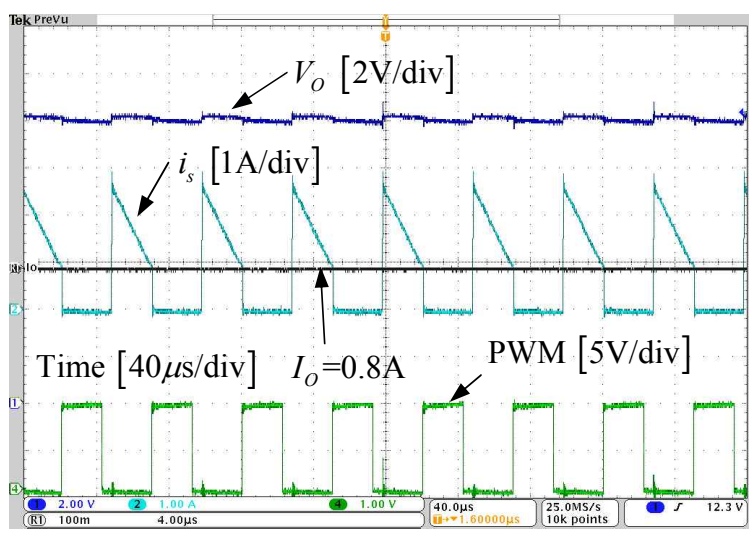

(b)

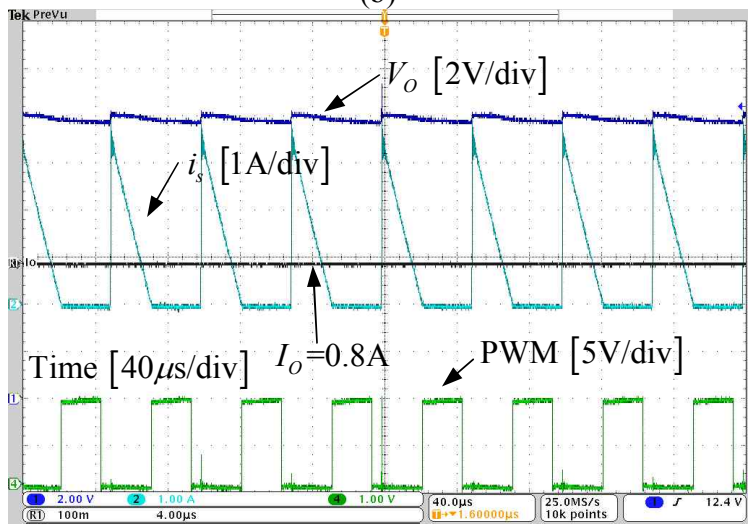

(d)

Fig. 9. The experimental waveforms of Flyback converter in different operation modes. (a) CISM-CCM. (b) Critical inductance state. (c)IISM-CCM. (d)IISM-DCM.

signal varies from 0 to $7 \mathrm{~V}$. The experimental waveforms are shown in Fig. 9(a), (b), (c) and (d). At the top of every diagram are the output voltages $u_{O}$ (deep blue). The middle waveforms are the secondary windings current $i_{s}$ (light blue) and the output current $I_{O}$ (black). At the bottom are the duty cycles (green). Obviously, the system operation modes fall in different regions with varied secondary self-inductances resulting from a distinct Magnetizing inductance $L_{M}$. Owing to the parasitic parameters of the electric component, the experimental waveforms show small differences when compared with the theoretical analysis.

In sum, the Flyback converter operates in three different modes: CISM-CCM, IISM-CCM and IISM-DCM. Meanwhile, the OVR in the CISM-CCM is the smallest among the three operation modes. $L_{s K}$ is the minimum secondary self-inductance that ensures the smallest OVR.

\section{CONCLUSIONS}

1) The paper divided the operation modes of an intrinsic safety Flyback converter into three types, namely, CISM-CCM, IISM-CCM and IISM-DCM. The expressions of the critical inductance among the three modes are $L_{s C}=R(1-d)^{2} / 2 f$ and

$$
L_{s K}=R(1-d)^{2} / 2 d f .
$$

2) The critical secondary self-inductance $L_{S K}$ is the minimum one that ensures that the OVR is smallest in the whole operation region, as well as the optimal parameter for the intrinsic safety Flyback converter.

3) After figuring out the OVRs in all kinds of operation modes and combining them with the critical inductances $L_{S C}$ and $L_{s K}$, they have a guiding significance for the design of the intrinsic safety system.

4) The IISM-DCM mode is not suitable for the intrinsic safety Flyback converter with a light resistance in high power, since the peak magnetizing current of the primary winding is $k$ times the secondary self-inductance.

5) This research helps the smaller secondary self-inductance and capacitance to meet the performance indexes in a given working scope. Meanwhile, it is constructive in terms of the miniaturization and integration design of the converter. In particular, the proposed method helps in designing the Flyback converter to meet the intrinsic safety characteristics, which are suitable 
for dangerous situation applications.

\section{REFERENCES}

[1] R. W. Erickson and D. Maksimovic, Fundamentals of Power Electronics, Springer, 2001.

[2] J. M. Adams, "Electrical apparatus for flammable atmospheres: Intrinsic safety," Power Eng. J., Vol. 5, No. 6, pp. 278-282, Nov. 1991.

[3] S. Liu, J. Liu, H. Mao, and Y. Zhang, "Analysis of operating modes and output voltage ripple of boost DC-DC converters and its design considerations," IEEE Trans. Power Electron., Vol. 23, No. 4, pp. 1813-1821, Jul. 2008.

[4] V. S. Nguyen, V. L. Tran, W. Choi, et al., "Analysis of the output ripple of the DC-DC boost charger for Li-Ion batteries," Journal of Power Eletronics, Vol. 14, No. 1, pp. 135-142, Jan. 2014

[5] S. Liu, J. Liu, Y. Yang, and J. Zhong, "Design of intrinsically safe buck dc-dc converters," in Proc. ICEMS'05, pp. 1327-1331, 2005.

[6] S. Liu, J. Liu, and H. Mao, "Analysis and design of boost dc-dc converters for intrinsic safety," in Proc. IPEMC'06, pp. 1267-1272, 2006.

[7] E. Babaei, M. E. S. Mahmoodieh, and H. M. Mahery, "Operational modes and output-voltage-ripple analysis and design considerations of buck-boost DC-DC converters," IEEE Trans. Ind. Electron., Vol. 59, No.1, pp. 381-391, Jan. 2012.

[8] E. Babaei and M. E. S. Mahmoodieh, "Calculation of output voltage ripple and design considerations of SEPIC converter," IEEE Trans. Ind. Electron., Vol. 61, No. 3, pp. 1213-1222, Mar. 2014

[9] A. Abramovitz, C. Liao and K. Smedley, "State-plane analysis of regenerative snubber for flyback converters," IEEE Trans. Power Electron., Vol. 28, No. 11, pp. 5323-5332, Nov. 2013.

[10] T. Bhattacharya, V. S. Giri, K. Mathew, and L. Umanand, "Multiphase bidirectional flyback converter topology for hybrid electric vehicles," IEEE Trans. Ind. Electron., Vol. 56, No. 1, pp. 78-84, Jan. 2009.

[11] G. M. L. Chu, D. D. C. Lu, and V. G. Agelidis, "Practical application of valley current mode control in a flyback converter with a large duty cycle," IET Power Electron., Vol. 5, No. 5, pp. 552-560, May 2012.

[12] J. Zhang, X. Huang, X. Wu, and Z. Qian, "A high efficiency flyback converter with new active clamp technique," IEEE Trans. Power Electron., Vol. 25, No. 7, pp. 1775-1785, Jul. 2010.

[13] J. Lee, "Single-stage AC/DC converter with input-current dead-zone control for wide input voltage ranges," IEEE Trans. Ind. Electron., Vol. 54, No. 2, pp. 724-732, Apr. 2007.

[14] S. H. Kang, D. Maksimovic, and I. Cohen, "Efficiency optimization in digitally controlled flyback DC-DC converters over wide ranges of operating conditions," IEEE Trans. Power Electron., Vol. 27, No. 8, pp. 3734-3748, Aug. 2012.

[15] S. Howimanporn and C. Bunlaksananusorn, "Performance comparison of continuous conduction mode (CCM) and discontinuous conduction mode (DCM) flyback converters," in PEDS, pp. 17-20, 2003.

[16] k. Billings and T. Morey, Switchmode Power Supply Handbook 3/E, McGraw-Hill, 2010
[17] M. Telefus, A. Shteynberg, M. Ferdowsi, and A. Emadi, "Pulse train control technique for flyback converter," IEEE Trans. Power Electron., Vol. 19, No. 3, pp. 757-764, May 2004.

[18] A. F. Witulski, "Introduction to modeling of transformers and coupled inductors," IEEE Trans. Power Electron., Vol. 10, No. 3, pp. 349-357, May 1995.

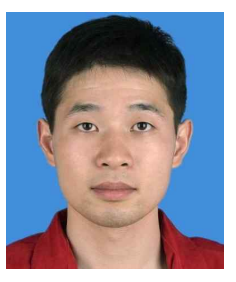

Wei Hu was born in Ningxiang, China, in 1980. He received his B.S. and M.S. degrees in the College of Electrical and Information Engineering, Hunan University, Changsha, China, in 2003 and 2006, respectively. He is currently working toward his Ph.D. degree. Since 2006, he has been with Guangzhou University, Guangzhou, China, where he is presently an Experimentalist in the Lab Center. His current research interests include the modeling and non-linear control of power converters, and stability analysis of DC-DC converters.

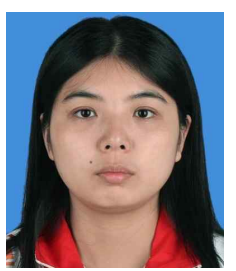

Fangying Zhang was born in Hubei, China, in 1981. She received her B.S. degree in Electronics and Information Engineering from the Hubei University of Technology, Wuhan, China, in 2003, and her M.S. degree in Geodetic Survey from Wuhan University, Wuhan, China, in 2006. She is presently an Experimentalist in the Lab Center of Guangzhou University, Guangzhou, China. Her current research interests include the modeling and non-linear control of power converters, and stability analysis of DC-DC converters.

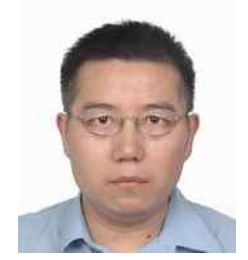

Yawu Xu was born in Hubei, China, in 1967. He received his B.S. and M.S. degrees, in 2003 and 2006, respectively. He is presently working toward his Ph.D. degree. His current research interests include the modeling and non-linear control of power converters, and stability analysis of DC-DC converters.

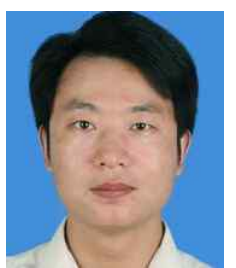

Xinbing Chen was born in Henan, China, in 1978. His current research interests include the modeling and non-linear control of power converters, and stability analysis of DC-DC converters. 